maximum quantities, the percentage of this quantity was calculated.

Results A total of 12 patients aged between one and 14 years were included. All children met adjusted parenteral recommendations for water soluble vitamins except for vitamin C, where intake was suboptimal in one case. In all patients, vitamin A recommendations were exceeded, whereas vitamins D and $\mathrm{K}$ were suboptimal. Recommendations for zinc were clearly surpassed in all children, but this was below the maximum quantity advised. Iodine was adequately supplied in $50 \%$, copper in $67 \%$ and selenium in $75 \%$ of patients. No patient exceeded the maximum recommended intake of vitamin $\mathrm{E}$ and chromium. The maximum dose of manganese was exceeded in one patient.

Conclusion Licensed parenteral multivitamin/multitrace products in the UK provide fixed combinations of multiple micronutrients and are dosed based on weight, although recommended intakes are mostly expressed as fixed daily quantities. Consequently, meeting the ESPHAN recommendations with these products is challenging and monitoring of serum concentrations of these nutrients is essential in this vulnerable patient population.

\section{P38 PEDIATRIC INFLAMMATORY BOWEL DISEASE AND HIDRADENITIS SUPPURATIVE: A CHALLENGING ASSOCIATION?}

Mark Mahon, Daniela Levanon. Jacobi Medical Center/Albert Einstein

10.1136/flgastro-2021-bspghan.47

Introduction Within Inflammatory Bowel Disease (IBD), perineal lesions are a common extra-intestinal manifestation, yet may mask other entities. Including several etiologies across a number of subspecialties, most of which are better appreciated in adulthood. This report focuses on an unusual dermatological association with IBD, presenting at an atypical time in the disease course.

Case Report 11-year-old obese Hispanic female presented with the chief complaint of epistaxis and was noted to have painful lower extremity nodules consistent with erythema nodosum and gluteal cleft lesions. Review of systems revealed fatigue, anorexia and diarrhea for two months prior accompanied by a $6.8 \mathrm{~kg}$ weight loss over that period. On admission, she was febrile $\left(38.3^{\circ} \mathrm{C}\right)$, tachycardic and hypotensive with baseline laboratory values notable for leukocytosis (13.1/ $\mathrm{nL}$ ), hypochromic microcytic anemia (hemoglobin $10.3 \mathrm{~g} / \mathrm{dL}$ and mean corpuscular volume $70.3 \mathrm{fL}$ ), thrombocytopenia $(55 / \mathrm{nL})$ and evidence of systemic inflammation with elevated CRP/ESR.

Stool studies were significant for fecal leukocytes and a CT revealed mural thickening with fat stranding; segmental colitis was confirmed on colonoscopy. At that time, a punch biopsy of the gluteal lesion revealed granulomatous dermatitis, presumed to be cutaneous Crohn's Disease (CD). Treatment was initiated with Metronidazole and Methylprednisolone. Soon after the clinical course became complicated by the development of a rectovaginal fistula. Induction and maintenance treatment were achieved with Infliximab and the patient was discharged with the diagnosis of CD with perineal involvement.

Multidisciplinary team monitoring over the following three years ensued before the patient reported progression of intertriginous lesions, this time to the axillae and infra-mammary areas. Punch biopsy at the new lesion established the diagnosis of Hidradenitis Suppurativa (HS), with a pathological confirmation.

Discussion HS is a chronic inflammatory dermatological disease of the apocrine glands, characterized by recurrent and painful, deep-seated nodules, abscesses, sinus tracts and/or fistulas. It affects inverse areas of the skin following the distribution of apocrine glands. Prevalence is higher post-puberty, with smoking and obesity acting as risk factors. The association with IBD, particularly $\mathrm{CD}$ is stronger in the severe phenotype and in pancolitis. The formal diagnosis is made on average one decade after the onset of IBD. Up to $25 \%$ of IBD patients experience extra-intestinal manifestations, perineal pathology accounts for $50 \%$ of the cases. Yet, in the absence of extra-perineal intertriginous involvement, the possibility of HS may be less recognizable. Improved awareness to this association among Paediatricians and Paediatric Gastroenterologists is important as co-pathology may require treatment escalation to immunosuppressive agents or alterations to monoclonal antibody regimen. More intensive treatment is often required as disease remission is harder to achieve for HS than CD.

Conclusion HS, when associated with CD, typically occurs one decade after the initial IBD diagnosis. This case presents a much shorter interval between such diagnoses, and potentially a dual presentation. This has not been appreciated in the literature to date and possibly suggests rare but earlier association when present. Analysis of a larger pediatric IBD cohort with HS will help clarify the pattern of association.

\section{P39 PHENOTYPE FLIP - RISK OF DEVELOPING CROHN'S DISEASE FOLLOWING RESTORATIVE PROCTO- COLECTOMY FOR ULCERATIVE COLITIS}

Alison Campbell, Bruce Jaffray. Great North Children's Hospital, Newcastle

\subsection{6/flgastro-2021-bspghan.48}

Introduction $30 \%$ of Ulcerative Colitis (UC) patients require surgery. Restorative procto-colectomy (RPC) with ileal pouch-anal anastomosis (IPAA) is the preferred procedure. Published experience suggests up to $25 \%$ of patients subsequently manifest Crohn's Disease (CD), and 50\% of these require pouch excision. Despite long-term follow up, we had not previously identified Crohn's conversion in our UC pouch patients.

Aim We analysed our UC cohort, for cases where the pouch was either excised or de-functioned. The aim of this study was to quantify the incidence of $\mathrm{CD}$ in this group.

Methods All children undergoing pouch surgery have had data regarding their surgery, pre-op management and disease status recorded contemporaneously. This database was interrogated and further results from the histology database retrieved. In addition, pre-pouch surgery work up, specifically diagnosis and number of pre-op colonoscopies, was evaluated.

Results From 1999 to 2020, 84 children (mean age 13.5 yrs) have undergone surgery for UC with the intention of performing RPC and IPAA. 3 were unable to be anastomosed at initial surgery; 1 subsequently underwent successful IPAA, 2 have end ileostomies. 